\title{
Positively versus negatively charged excitons: A high magnetic field study of $\mathrm{CdTe} / \mathrm{Cd}_{1-x} \mathrm{Mg}_{x} \mathrm{Te}$ quantum wells
}

\author{
G. Bartsch, ${ }^{1}$ M. Gerbracht, ${ }^{1}$ D. R. Yakovlev, ${ }^{1,2}$ J. H. Blokland, ${ }^{3}$ P. C. M. Christianen, ${ }^{3}$ E. A. Zhukov, ${ }^{1,4}$ A. B. Dzyubenko, ${ }^{5}$ \\ G. Karczewski, ${ }^{6}$ T. Wojtowicz, ${ }^{6}$ J. Kossut, ${ }^{6}$ J. C. Maan, ${ }^{3}$ and M. Bayer ${ }^{1}$ \\ ${ }^{1}$ Experimentelle Physik 2, Technische Universität Dortmund, D-44221 Dortmund, Germany \\ ${ }^{2}$ A. F. Ioffe Physical-Technical Institute, Russian Academy of Sciences, 194021 St. Petersburg, Russia \\ ${ }^{3}$ High Field Magnet Laboratory, Institute for Molecules and Materials, Radboud University Nijmegen, NL-6525 ED Nijmegen, \\ The Netherlands \\ ${ }^{4}$ Faculty of Physics, M.V. Lomonosov Moscow State University, 119992 Moscow, Russia \\ ${ }^{5}$ Department of Physics, California State University at Bakersfield, Bakersfield, California 93311, USA \\ ${ }^{6}$ Institute of Physics, Polish Academy of Sciences, PL-02668 Warsaw, Poland \\ (Received 8 February 2011; revised manuscript received 12 April 2011; published 10 June 2011)
}

\begin{abstract}
A magneto-optical study of the energy and spin structure of charged excitons in a 20-nm-thick $\mathrm{CdTe} / \mathrm{Cd}_{0.65} \mathrm{Mg}_{0.35} \mathrm{Te}$ quantum well is performed in strong magnetic fields up to $51 \mathrm{~T}$. The type of resident carriers (holes or electrons) in the quantum well is controlled optically by above-barrier illumination, permitting a direct comparison of positively $\left(\mathrm{T}^{+}\right)$versus negatively $\left(\mathrm{T}^{-}\right)$charged excitons. The binding energies of the singlet states of these complexes behave qualitatively differently with increasing magnetic field $B$; namely, the binding energy decreases for $\mathrm{T}^{+}$and increases for $\mathrm{T}^{-}$with $B$. The triplet state of $\mathrm{T}^{+}$is identified in strong fields with a binding energy smaller than that of the $\mathrm{T}^{-}$triplet state.
\end{abstract}

DOI: 10.1103/PhysRevB.83.235317

PACS number(s): 78.55.Et, 73.21.Fg, 78.67.De, 71.35.Pq

\section{INTRODUCTION}

Charged excitons, or trions, are semiconductor exciton complexes made up of three carriers. A negatively charged exciton, $\mathrm{T}^{-}$, is a bound state of two electrons and one hole, while a positively charged exciton, $\mathrm{T}^{+}$, consists of two holes and one electron. In bulk three-dimensional semiconductors free trions are very loosely bound and have never been identified unambiguously. However, reduction of dimensionality enhances the trion binding, which makes free trions much more robust. In fact, while predicted theoretically for bulk systems by Lampert in $1958,{ }^{1}$ negatively charged trions have been observed experimentally in 1993, more than three decades later, in $\mathrm{CdTe} /(\mathrm{Cd}, \mathrm{Zn}) \mathrm{Te}$ quantum wells $(\mathrm{QWs}){ }^{2}$ Note that an external magnetic field $B$ and/or additional carrier spatial confinement (and localization) may further increase trion binding.

Charged trions are now commonly observed in semiconductor QWs. The negatively charged species $\mathrm{T}^{-}$are studied in detail in various material systems, such as $\mathrm{GaAs} /(\mathrm{Al}, \mathrm{Ga}) \mathrm{As},{ }^{3-5}$ CdTe-based, ${ }^{6,7}$ and ZnSe-based ${ }^{8-10}$ structures. Magnetooptical techniques play a key role in these investigations and allow one to obtain comprehensive information about the energy and the spin structure of the $\mathrm{T}^{-}$complex. In particular, it is shown that additionally to the ground trion state, which is an electron singlet, the trion triplet states are bound at finite magnetic fields. ${ }^{5,11-13}$ Two types of triplet states-dark and bright-are studied theoretically ${ }^{14-18}$ and experimentally. ${ }^{7,19,20}$ In magnetic fields above $20 \mathrm{~T}$ the binding energy of the triplet state, which is dark, may exceed the singlet state binding energy, so that the triplet state is the ground state of $\mathrm{T}^{-}$. This depends sensitively on the signs and values of the electron and hole Zeeman splittings and on the details of the quasi-2D confinement in the QW (QW depth and width, effective masses of the carriers). Such a singlet-triplet crossover can either be observed directly, as a crossing of the corresponding optical lines, e.g., in emission spectra, or it may be hidden and affect only the intensities of these transitions. ${ }^{7}$ A comprehensive spectroscopic study, involving the analysis of circularly polarized emission, absorption, and reflectivity spectra is required, in order to get a reliable separation of the Coulomb and Zeeman contributions to the trion binding energies. Such an analysis is hampered by the fact that the hole Zeeman splitting in QWs is typically a nonlinear function of the magnetic field strength and, as such, is not described by a field-independent $g$ factor, as shown below.

Positively charged trions $\mathrm{T}^{+}$are observed in different $\mathrm{QW}$ systems as well: GaAs/(Al,Ga)As, ${ }^{5,12,21-24} \mathrm{CdTe}$-based, ${ }^{25-27}$ and $\mathrm{ZnSe} /(\mathrm{Zn}, \mathrm{Mg})(\mathrm{S}, \mathrm{Se})$ structures. ${ }^{8,10}$ The triplet $\mathrm{T}_{t}^{+}$state is stabilized in high magnetic fields. ${ }^{12,21,22,24}$ Comparative studies of negatively and positively charged excitons in the very same QW, where the type of resident carrier is tuned either by a gate voltage or by above-barrier illumination, have been reported for $\mathrm{GaAs} /(\mathrm{Al}, \mathrm{Ga}) \mathrm{As}^{12,23}$ and $\mathrm{ZnSe} /(\mathrm{Zn}, \mathrm{Mg})(\mathrm{S}, \mathrm{Se})^{10}$ QW structures.

Experimentally, it is established that at zero magnetic field, $B=0$, the binding energies of the singlet trions $\mathrm{T}_{s}^{-}$and $\mathrm{T}_{s}^{+}$are comparable, with about $10 \%$ smaller values for the $\mathrm{T}^{+}$. This may appear quite counterintuitive, because larger binding energies are generally expected in quantum mechanics for complexes composed of heavier particles. However, the unexpectedly very close values of the $\mathrm{T}^{-}$and $\mathrm{T}^{+}$binding energies are confirmed by theoretical calculations within a simple valence band model. ${ }^{28,29}$ Note though that complications arising from the complex structure of the valence band may be quantitatively important. ${ }^{30}$

The magnetic field dependencies of the $\mathrm{T}^{-}$and $\mathrm{T}^{+}$binding energies differ considerably. Namely, while the binding energy of the $\mathrm{T}_{s}^{-}$singlet state increases with the field, it decreases for the $\mathrm{T}_{s}^{+}$. In both cases the binding energies saturate in high magnetic fields, typically above $20 \mathrm{~T}$. Also, the appearance 
of trion triplet states in the optical spectra with increasing $B$ is quite different. To the best of our knowledge, $\mathrm{T}_{t}^{+}$ triplet states in CdTe-based QWs have not been studied so far.

In this paper we present a comparative study of the $\mathrm{T}^{+}$and $\mathrm{T}^{-}$spin and energy structures in a $\mathrm{CdTe} /(\mathrm{Cd}, \mathrm{Mg}) \mathrm{Te}$ quantum well. We perform a detailed analysis of the experimental data obtained by photoluminescence (PL), PL excitation (PLE), and reflectivity measurements. The type of resident carrier is changed, from holes to electrons, by application of different illumination conditions. The magneto-optical properties of the positively and negatively charged excitons are studied. This allows us to identify the singlet and triplet trion states for both $\mathrm{T}^{+}$and $\mathrm{T}^{-}$, and to extract their magnetic field-dependent Coulomb binding energies.

The paper is organized as follows. Experimental details are given in Sec. II. In Sec. III we consider the spin structures of $\mathrm{T}^{+}$and $\mathrm{T}^{-}$and the polarization of their optical transitions in absorption and emission. Methods for the optical control of the carrier concentration and the identification of the carrier type are introduced here. Experimental results of magneto-optical studies of trions in high magnetic fields up to $51 \mathrm{~T}$ are given in Sec. IV. There we separate the effects of the external magnetic field on the diamagnetic shift, on Zeeman splittings, and on the Coulomb energies of the trion states. Furthermore, the trion polarization in reflection and emission spectra is discussed. We present the observation of the $\mathrm{T}^{+}$triplet state and finally focus on the comparative study of the Coulomb energies of the $\mathrm{T}^{+}$and $\mathrm{T}^{-}$trions. In Sec. $\mathrm{V}$ conclusions are drawn.

\section{EXPERIMENTALS}

The sample studied is a 20 -nm-thick $\mathrm{CdTe} / \mathrm{Cd}_{0.63} \mathrm{Mg}_{0.37} \mathrm{Te}$ single quantum well structure (sample code 090505AC), grown by molecular-beam epitaxy on a (100)-oriented GaAs substrate. The top barrier separating the QW from the surface has a thickness of $135 \mathrm{~nm}$. The sample is not intentionally doped, but due to residual impurities it is slightly $p$-type doped, leading to a two-dimensional hole gas (2DHG) with a density ${ }^{31}$ lower than $10^{10} \mathrm{~cm}^{-2}{ }^{32}$ Illumination with photon energies higher than the band gap of the $\mathrm{Cd}_{0.63} \mathrm{Mg}_{0.37}$ Te barriers (above $2.26 \mathrm{eV}$ ) inverts the type of resident carriers in the QW. A two-dimensional electron gas (2DEG) with a density of a few $10^{9} \mathrm{~cm}^{-2}$ can be achieved by these means. We use this method to perform a comparative study of the positively and negatively charged excitons in the same sample, similar to Ref. 10, where $\mathrm{ZnSe} /(\mathrm{Zn}, \mathrm{Mg})(\mathrm{S}, \mathrm{Se}) \mathrm{QW}$ have been investigated. $\mathrm{T}^{+}$and $\mathrm{T}^{-}$ can be distinguished in the optical spectra directly, by their different polarization properties in external magnetic fields. This allows a straightforward comparison of the parameters for the different charged excitons under identical confinement and localization conditions.

Photoluminescence, PL excitation, and reflectivity spectra are measured in high magnetic fields applied parallel to the structure growth axis (Faraday geometry, $\mathbf{B} \| \mathbf{z}$ ). A continuous-wave tunable Ti:Sph laser and a halogen lamp are used as light sources. Signals are detected by a liquidnitrogen-cooled silicon charge-coupled-device (CCD) detector. Magnetic fields are generated either by a superconducting solenoid (17 T) or by a resistive Florida-Bitter magnet (33 T).
Stronger fields up to $51 \mathrm{~T}$ are achieved using a pulsed magnet. The experiments are performed at low temperatures, ranging from 0.4 to $4.2 \mathrm{~K}$. For most experiments the optical excitation and detection is provided by optical step index fibers, but in some special cases, noted in the text, we use direct optical access to the sample via cryostat windows. Polarizers are implemented between the sample and the fibers to analyze the degree of circular polarization. All optical spectra in this article are corrected for small polarization leakages that occur due to imperfections in the polarizers.

Additionally we use a time-resolved pump-probe Kerr rotation technique, which is commonly applied for the investigation of coherent spin dynamics of carriers. ${ }^{34}$ In this paper it permits the identification of the type of resident carrier in the QW and measuring the electron $g$ factor. Further details on the application of this technique to $\mathrm{CdTe} /(\mathrm{Cd}, \mathrm{Mg}) \mathrm{Te} \mathrm{QWs}$ can be found in Refs. 32, 35 and 36.

\section{IDENTIFICATION OF TRIONS AND RESIDENT CARRIERS}

The type of trion depends on the type of resident carrier in the QW. Resident electrons and holes lead to photogeneration of $\mathrm{T}^{-}$and $\mathrm{T}^{+}$, respectively. Commonly, magnetotransport techniques are used to identify carrier types and their concentration and mobility. Being well suited for modulationdoped structures with relatively high carrier concentrations these techniques are, however, not suitable for QWs with low carrier densities. The problem is especially evident at low temperatures, when the in-plane carrier motion becomes affected by potential fluctuations, caused by variations in the QW width and the barrier alloy.

Optical methods are established to be the most reliable technique for weakly doped structures, where the resident carriers are localized and cannot be addressed by transport techniques. ${ }^{32,38}$ Pump-probe Kerr or Faraday rotation techniques give direct access to the properties of the resident carriers, also providing their $g$ factor values and spin dephasing times. ${ }^{34}$ Even the coexistence of resident electrons and holes, localized in different sites of the same QW, can be identified by these techniques. ${ }^{32}$ Magneto-optical PL and reflectivity studies provide rich information about neutral and charged excitons, including Coulomb binding energies, diamagnetic shifts, Zeeman splittings, and polarization properties. Altogether this allows a precise identification of the different types of trions within the different regimes, as will be shown in the following section.

\section{A. Schematics for the trion spin structure in magnetic field}

Let us first consider the schematics of the trion spin structure in external magnetic fields and analyze the selection rules and polarizations for allowed optical transitions. There is a certain freedom in the definition of the $g$ factors ${ }^{39}$ of free carriers, excitons, and trions. Our definition of the $g$ factor is chosen to provide maximal universality. Note that one can find several different systems of trion $g$ factor terminology in the literature, which actually describe the same physics. In this study we restrict ourselves to heavy-hole states only and use a convention based on the following conditions: 
(1) The electron representation is used; i.e., both electrons and holes have the same energy axis, whose values increase from bottom to top. The same is true for exciton and trion complexes.

(2) A universal definition for the $g$-factor sign is used: For $g>0$ the spin-down state with negative spin sign is the lowest in energy (i.e., $-1 / 2$ for electrons, $-3 / 2$ for heavy-holes, -1 for excitons, $-3 / 2$ for negatively charged singlet trions, and $-1 / 2$ for positively charged singlet trions).

(3) The Zeeman splittings of the free carriers, excitons, and singlet trions are described in the same universal (i.e., spin independent) form: $\Delta E_{i}=\mu_{B} g_{i} B$, where $i$ is the index of the respective object and $\mu_{B}$ is the Bohr magneton.

(4) The exciton spin Hamiltonian, consisting of the electron and heavy-hole parts, reads

$$
H_{X}=\left(g_{e} S+\frac{g_{h}}{3} J\right) \mu_{B} B,
$$

where $g_{e}$ and $g_{h}$ are the $g$ factors for electrons and holes, $S=$ $\pm 1 / 2$ is the electron spin, and $J= \pm 3 / 2$ is the hole magnetic moment, which is often noted as the heavy-hole spin. It follows from this Hamiltonian that the $g$ factor for the bright exciton with spin \pm 1 is

$$
g_{X}=g_{h}-g_{e} .
$$

(5) Spin allowed optical transitions satisfy the selection rule $\Delta S= \pm 1$, where the magnetic moment \pm 1 is introduced or removed by circular polarized photons with $\sigma^{+}$and $\sigma^{-}$ polarizations, respectively.

Our convention is quite similar to the one used by van Kesteren et al., ${ }^{40}$ which is widely applied for semiconductor QWs. The only difference is that the sign of the $g$ factors for the heavy-hole, exciton, and negatively charged trion are reversed. It results, respectively, in a different definition of the $g$ factor for the bright exciton: $g_{X}=g_{e}+g_{h}$; compare with Eq. (2).

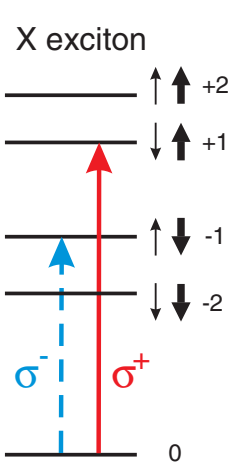

$$
\text { n-type }
$$

$$
\mathrm{T}_{\mathrm{s}}{ }^{-} \text {singlet trion }
$$
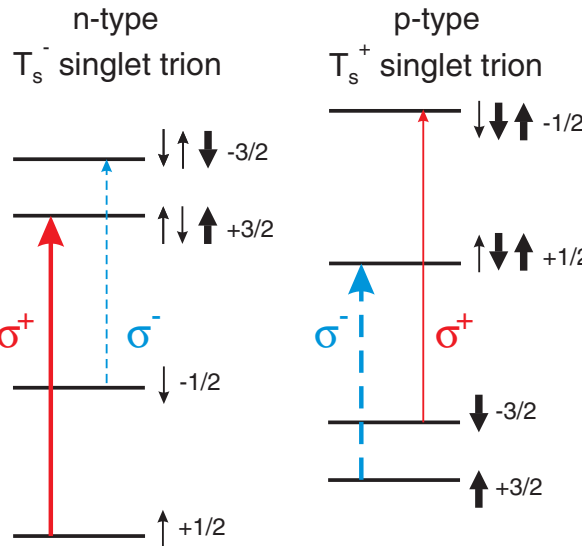

FIG. 1. (Color online) Scheme (ABSORPTION) of the spin structure and optical transitions for excitons and $\mathrm{T}_{s}^{-}$and $\mathrm{T}_{s}^{+}$singlet trions in external magnetic fields. Used parameters: $g_{e}<0, g_{h}<0$, and $\left|g_{e}\right|>\left|g_{h}\right|$. Short black arrows show carrier spins: thin for electrons and thick for holes. They are aligned up and down for + and - spin sign, respectively. The total spin of each state is additionally given by numbers. The thickness of the colored arrows is proportional to the probability of the optical transitions.

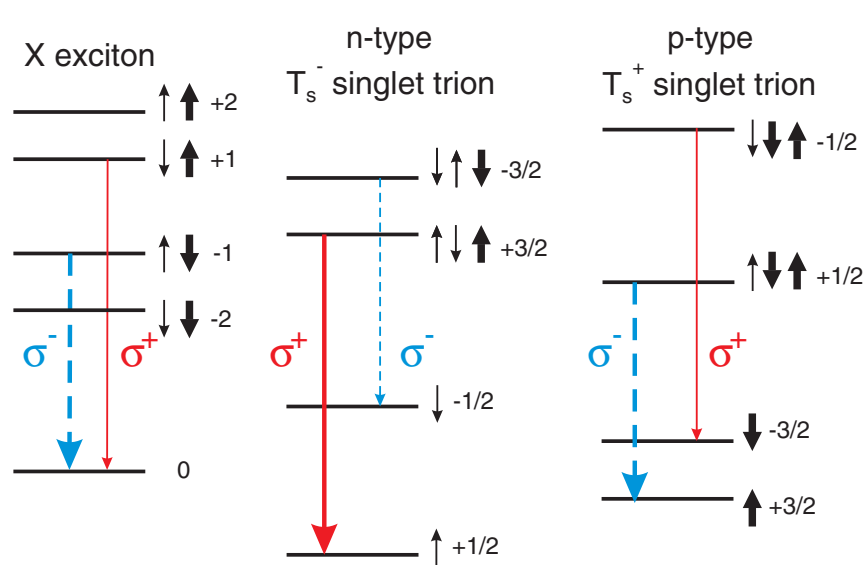

FIG. 2. (Color online) Scheme (EMISSION) of the spin structure and optical transitions for excitons and $\mathrm{T}_{s}^{-}$and $\mathrm{T}_{s}^{+}$singlet trions in external magnetic fields. Used parameters: $g_{e}<0, g_{h}<0$, and $\left|g_{e}\right|>\left|g_{h}\right|$.

The variety of spin schematics for singlet and triplet trion states is controlled by the signs of the electron and hole $g$ factors and their mutual size. To exemplify the schematics in Figs. 1, 2, and 3 we choose $g_{e}<0, g_{h}<0$, and $\left|g_{e}\right|>\left|g_{h}\right|$, which correspond to the conditions of the QW studied in this article.

In Fig. 1 the optical selection rules for the absorption (i.e., photoexcitation) of excitons and singlet states of $\mathrm{T}^{-}$and $\mathrm{T}^{+}$ are analyzed in the limit of low lattice temperatures $\left(k_{B} T<\right.$ $\left.\Delta E_{i}\right)$. Under this condition only the lowest energy spin states are populated. As a result, the absorption probabilities of the respective optical transitions, coded by the arrow thickness in the scheme, depend on the thermal population of the resident carriers of the Zeeman levels. The exciton absorption is equal for both circular polarizations, because the initial state, which
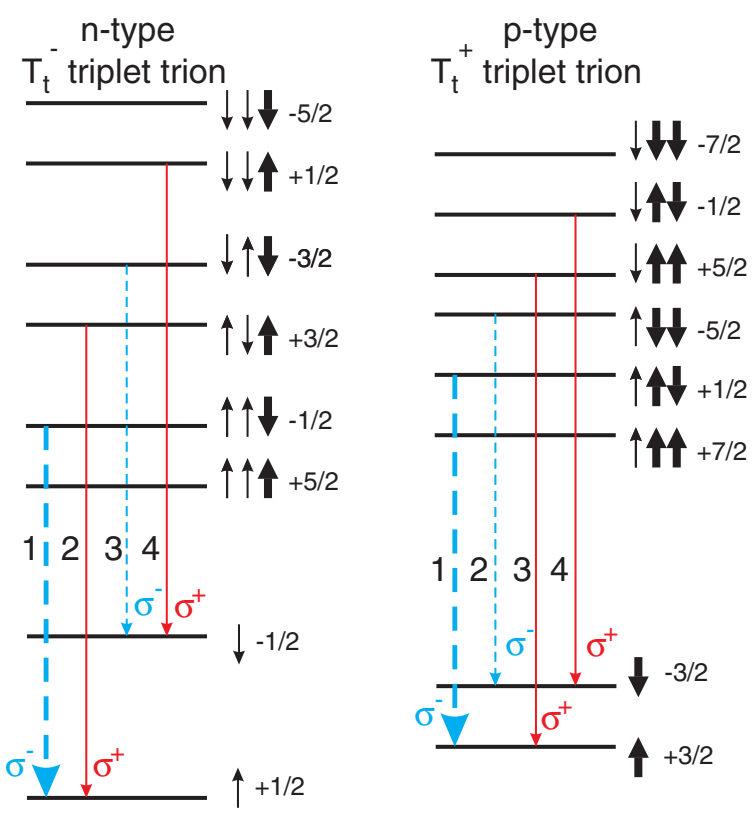

FIG. 3. (Color online) Triplet trion schemes and optical transitions (EMISSION) for the $n$-type and $p$-type regimes. Used parameters: $g_{e}<0, g_{h}<0$, and $\left|g_{e}\right|>\left|2 g_{h}\right|$. 
is an unexcited crystal, is not polarized. The length of the optical transition arrows corresponds to the photon energy. Therefore, from this schematic the spin splitting of the optical transitions can be evaluated.

The schematic for the exciton and trion emission is given in Fig. 2. For the chosen set of parameters the optical transitions in emission are simply the reversed absorption transitions. A complete set of experimental data, consisting of the Zeeman splittings and polarizations of the trion optical transitions in absorption and emission, is required to determine the trion spin structure. An example of such an analysis for a 12-nm-thick $\mathrm{CdTe} / \mathrm{Cd}_{0.85} \mathrm{Mg}_{0.15} \mathrm{Te} \mathrm{QW}$ is given in Ref. 7. For the case considered here, one can see that both in emission and in absorption the optical transitions of $\mathrm{T}_{s}^{-}$and $\mathrm{T}_{s}^{+}$have opposite polarization (Figs. 1 and 2). This allows one to distinguish trions of opposite charge, and correspondingly, the type of resident carrier. Note that this is not a general rule for all possible trions and depends on the specific values for the electron and hole $g$ factors.

The schematics for the emission of $\mathrm{T}_{t}^{-}$and $\mathrm{T}_{t}^{+}$triplet trions are given in Fig. 3. Here an additional criterion is responsible for the ordering of the triplet spin states, namely, $\left|g_{e}\right|>\left|2 g_{h}\right|$. The strongest optically active transitions in emission are $\sigma^{-}$ polarized for both $\mathrm{T}_{t}^{+}$and $\mathrm{T}_{t}^{-}$. The schemes for the triplet states absorption can be received from the emission schematics by reversing the arrows. However the strongest transitions are determined by the spin state that is lowest in energy, i.e., $+1 / 2$ for electrons and $+3 / 2$ for heavy holes.

\section{B. Optical control of the type of resident carrier}

Photoluminescence spectra for the two different excitation regimes, above and below barrier illumination, are shown in Fig. 4 by a dashed and a solid line, respectively. The exciton line, with a maximum at $1.6017 \mathrm{eV}$, is clearly seen for abovebarrier excitation. The second and strongest line, shifted by $2.3 \mathrm{meV}$ to lower energies for both illumination conditions, is the trion emission. The trion binding energies for positively and negatively charged trions are nearly the same at $B=0 \mathrm{~T} .{ }^{10}$ Thus based on this spectrum alone, it is not possible to identify the charge of the trion complex. However, we show in Sec. IV

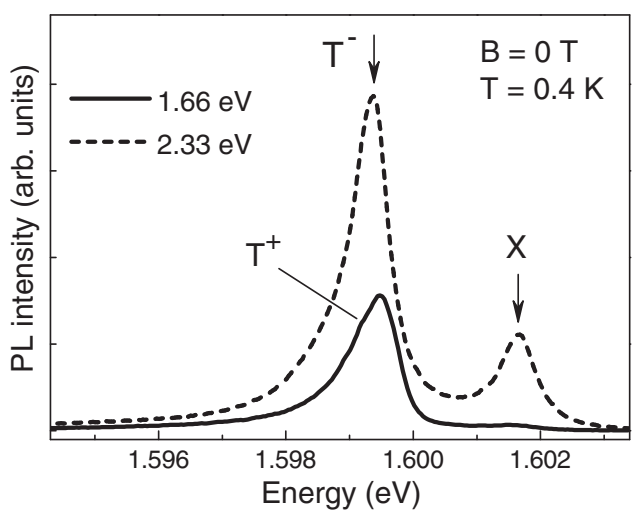

FIG. 4. Photoluminescence spectra of a 20-nm-thick $\mathrm{CdTe} / \mathrm{Cd}_{0.63} \mathrm{Mg}_{0.37} \mathrm{Te} \mathrm{QW}$ measured for optical excitation below $(1.66 \mathrm{eV})$ and above $(2.33 \mathrm{eV})$ the band gap of the $\mathrm{Cd}_{0.63} \mathrm{Mg}_{0.37} \mathrm{Te}$ barriers. The peaks are assigned to the exciton (X) and the positively $\left(\mathrm{T}_{s}^{+}\right)$and negatively $\left(\mathrm{T}_{s}^{-}\right)$charged singlet trions.

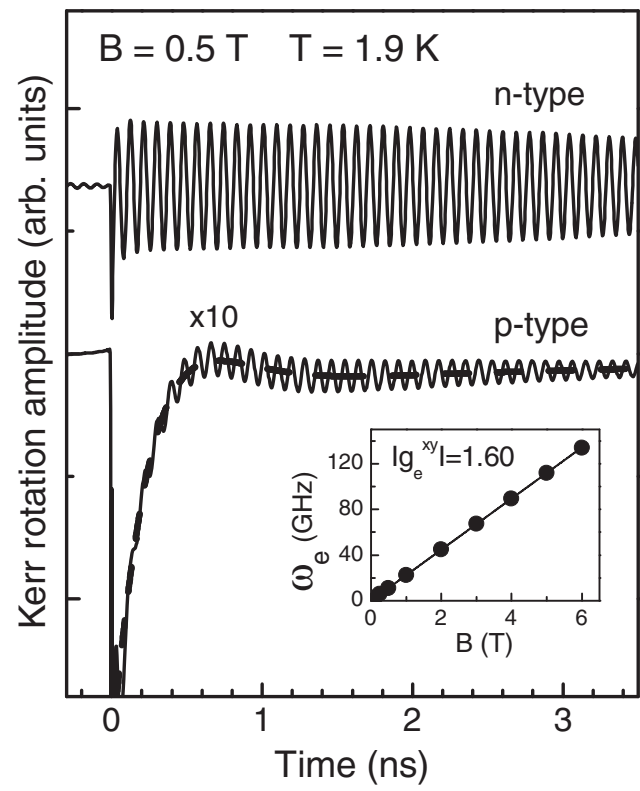

FIG. 5. Pump-probe Kerr rotation signals, measured for resonant excitation of the trion state with and without additional illumination at $2.33 \mathrm{eV}$, i.e. in the $n$-type and $p$-type regimes, respectively. The dashed line in the lower signal corresponds to a fit of the hole spin beats. The magnetic field is applied perpendicular to the growth axis of the QW structure (Voigt geometry). The inset shows the magnetic field dependence of the Larmor frequency of the electrons, which allows us to evaluate $\left|g_{e}^{x, y}\right|=1.60$. The $g$-factor component parallel to the growth axis, $\left|g_{e}^{z}\right|=1.70$, is evaluated by similar measurements at oblique angles of $3^{\circ}$.

that in external magnetic fields, the set of $g$ factors for the investigated sample results in opposite circular polarizations for the $\mathrm{T}^{-}$and the $\mathrm{T}^{+}$emission lines. By this method we show that under above-barrier photoexcitation the dominating recombination is due to excitons and $\mathrm{T}_{s}^{-}$trions, while under below-barrier excitation the strongest line at $1.5995 \mathrm{eV}$ is due to $\mathrm{T}_{s}^{+}$.

Clear evidence for the tunability of the types of resident carriers in the structure studied here comes from pumpprobe Kerr rotation experiments on the coherent carrier spin dynamics in external magnetic fields. The experimental data shown in Fig. 5 are measured using a laser photon energy tuned to the trion resonance. Additional above-barrier illumination at $2.33 \mathrm{eV}$ by a weak continuous-wave laser is used to control the type of the resident carrier. Note that under resonant excitation of trions their radiative recombination time in $\mathrm{CdTe} /(\mathrm{Cd}, \mathrm{Mg}) \mathrm{Te}$ QWs is less than $30-50$ ps. ${ }^{33,36}$ Therefore, at longer delays the Kerr rotation signal is generated by the resident carriers only. The Kerr rotation signal in the upper panel of Fig. 5, measured with above barrier illumination, is typical for $n$-doped QWs. ${ }^{34}$ It shows fast electron spin beats decaying with increasing delay. The signal is symmetric with respect to its zero level. Only a small asymmetry at very short delays $(<100 \mathrm{ps})$ is visible, which is typically assigned to photogenerated holes.

In the absence of above-barrier illumination the Kerr rotation signal is drastically different. The lower signal in Fig. 5 shows a single, strongly damped oscillation with a very large 
period. It can be assigned to the resident heavy holes with a very small value of their in-plane $g$ factor. One can also see a fast oscillating component in the signal, which is due to a small amount of resident electrons left in the system. These electrons are spatially separated in the QW plane and localized on QW width fluctuations at places where holes are absent. From the Kerr rotation signal we conclude that without above-barrier illumination resident holes and electrons coexist in the QW. The hole concentration is about 20 times larger than that of the electrons; for details see Ref. 31. As a result the $\mathrm{T}^{+}$emission is dominant in PL for below-barrier excitation.

The Kerr rotation signals can be fitted by the following equation:

$$
\begin{aligned}
A_{\mathrm{KR}}(t)= & A_{e} \exp \left(-\frac{t}{T_{2, e}^{*}}\right) \cos \left(\omega_{e} t\right) \\
& +A_{h} \exp \left(-\frac{t}{T_{2, h}^{*}}\right) \cos \left(\omega_{h} t\right) .
\end{aligned}
$$

Here

$$
\omega_{e(h)}=\frac{g_{e(h)} \mu_{B} B}{\hbar}
$$

is the Larmor frequency of the carrier spin precession about an external magnetic field $B . T_{2, e}^{*}$ and $T_{2, h}^{*}$ are spin dephasing times for electrons and holes, respectively. $A_{e}$ and $A_{h}$ are the electron and hole spin beat amplitudes.

The Kerr rotation signal in the $p$-type regime is fitted using the following parameters: $A_{e} / A_{h}=0.05, \omega_{e}=11.2 \mathrm{GHz}$, $\omega_{h}=0.84 \mathrm{GHz}, T_{2, e}^{*}=4.5 \mathrm{~ns}$, and $T_{2, h}^{*}=290 \mathrm{ps}$. The hole contribution to the signal is shown by the dashed lines in the lower panel of Fig. 5. The in-plane $g$ factor of the heavy holes is close to zero, which causes a very large period of the Larmor spin precession and strong damping, because the dephasing time is considerably shorter than the precession period.

Under above-barrier illumination the QW contains resident electrons. Compared to the $p$-type regime the amplitude of the electron Kerr rotation signal increases by a factor of 40 (note the scaling factor 10 for signal in the $p$-type regime). The long-living contribution (i.e., exceeding 100 ps) of the resident holes is absent in this signal. The fitting parameters for this case are $A_{e} / A_{h} \approx 1, \omega_{e}=11.2 \mathrm{GHz}, T_{2, e}^{*}=5.6 \mathrm{~ns}$, and $T_{2, h}^{*}=30 \mathrm{ps}$.

The inset in Fig. 5 shows the magnetic field dependence of the electron spin precession frequency. Its linear interpolation leads to a value of the in-plane electron $g$ factor $\left|g_{e}^{x, y}\right|=1.60$. The $g$-factor component $\left|g_{e}^{z}\right|=1.70$, which is essential for constructing the spin structure in the Faraday geometry, is evaluated from the Kerr rotation signals measured at a small deviation angle of $3^{\circ}$ from the Faraday geometry. ${ }^{37}$ In the main part of this paper we analyze the Zeeman splittings of excitons and trions in the Faraday geometry, and for simplicity we omit the index $z$ for $g$ factors.

\section{Reflectivity spectra in high magnetic fields}

The strong Coulomb interaction in II-VI semiconductors results in strong exciton resonances, which can be conveniently measured in reflectivity spectra. Measuring the polarizationresolved spectra in external magnetic fields provides rich



FIG. 6. (Color online) Reflectivity spectra at $B=14 \mathrm{~T}$ for two circular polarizations. The spectra are shifted vertically for better visibility. The polarization degree of the trion as a function of the magnetic field is shown in the inset by open circles. A fit using Eq. (5) (dashed line) reveals a hole temperature of $T_{h}=0.6 \mathrm{~K}$.

information about the energy and spin structure of excitons and trions.

Illustrative reflectivity spectra for the QW studied are given in Fig. 6 for an external magnetic field of $14 \mathrm{~T}$, applied in the Faraday geometry. Two circular polarizations, measured in the $p$-type regime at $T=0.4 \mathrm{~K}$, are shown. To avoid above-barrier illumination the halogen lamp is spectrally cut off at $1.70 \mathrm{eV}$ by a low-pass optical filter. The strongest resonance is identified as the $1 s$ heavy-hole $(\mathrm{hh})$ exciton $\mathrm{X}_{\mathrm{hh}, 1 s}$. In agreement with Fig. 1, its oscillator strength is the same in both polarizations. However the $\sigma^{-}$resonance is slightly broader than the $\sigma^{+}$ resonance, which is most probably due to exchange scattering with resident holes. ${ }^{9}$ The trion resonance is visible as a small peak in the $\sigma^{-}$polarization on the low energy side of the exciton. Being very pronounced in $\sigma^{-}$polarization it vanishes in $\sigma^{+}$polarization, which is in agreement with the scheme for the $p$-type case in Fig. 1. This allows us to identify this trion as $\mathrm{T}^{+}$.

In the energy range of $1.62-1.64 \mathrm{eV}$ in Fig. 6 four weaker resonances are visible. To identify them, we present the corresponding fan chart diagram in Fig. 7. Generally, the $1 s$ exciton states exhibit weak diamagnetic shifts with increasing magnetic field, irrespective of the electron or hole confinement levels. The $2 s$ exciton states, however, show a strong diamagnetic shift, e.g., for $\mathrm{X}_{\mathrm{hh}, 2 s}$. The following transitions can be assigned: $\mathrm{X}_{\mathrm{lh}, 1 s}, \mathrm{X}_{e 1-\mathrm{hh} 3}$, and $\mathrm{X}_{e 2-\mathrm{hh} 2}$. (The indexes $e, \mathrm{hh}$, and $\mathrm{lh}$ stand for electron, heavy hole, and light hole, respectively, where the numbers give the quantum confinement level. $1 s$ and $2 s$ refer to the respective exciton states.) The resonance of $\mathrm{T}^{+}$shifts roughly parallel with $\mathrm{X}_{\mathrm{hh}, 1 s}$, reflecting that the binding energy of positively charged trions has only a weak dependence on the magnetic field. Note, that the Zeeman splitting of $\mathrm{X}_{\mathrm{lh}, 1 s}$ considerably exceeds that of $\mathrm{X}_{\mathrm{hh}, 1 \mathrm{~s}}$. From the data of Fig. 7 the exciton $g$ factors and their 


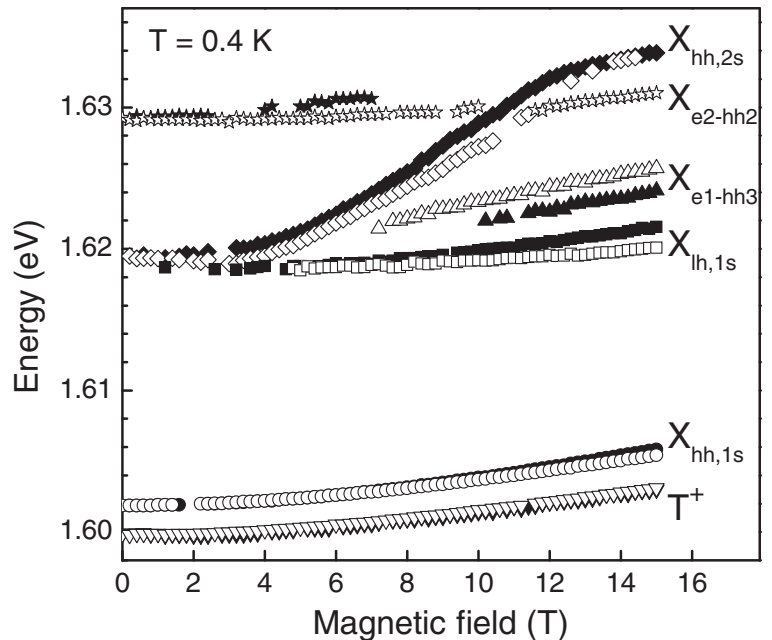

FIG. 7. Peak positions of the exciton transitions vs magnetic field strength evaluated from the reflectivity measurements. The symbols show resonances observed in $\sigma^{+}$(closed) and $\sigma^{-}$(open) polarizations.

magnetic field dependencies are evaluated and discussed in detail in Sec. IV C.

\section{MAGNETO-OPTICAL INVESTIGATIONS OF THE TRIONS}

In this section we focus on the detailed analysis of magnetoluminescence spectra, measured in both the $p$-type and $n$-type regimes. Our goal is to collect comprehensive information on the energy and spin structures of positively and negatively charged trions.

\section{A. Magnetoluminescence of positively and negatively charged trions}

In Fig. 8 we present photoluminescence spectra measured for two circular polarizations in a magnetic field of $15 \mathrm{~T}$. The two panels display results for the $p$-type and $n$-type doped regimes: In panel (a) $\mathrm{T}^{+}$is dominating, while in panel (b) $\mathrm{T}^{-}$plays the main role. For the $\mathrm{T}^{-}$regime singlet $\left(\mathrm{T}_{s}^{-}\right)$and triplet $\left(\mathrm{T}_{t}^{-}\right)$states are observed. ${ }^{7}$ For the $p$-type case only the singlet state $\left(\mathrm{T}_{s}^{+}\right)$is visible at $B=15 \mathrm{~T}$. All emission lines in both panels are strongly polarized. The singlet states of $\mathrm{T}^{+}$ and $\mathrm{T}^{-}$have opposite polarization, which is in agreement with the schematics in Fig. 2. The polarization of the peaks is used below to identify the trion states on the basis of a reconstruction of the trion fine structure.

A closer look at the energy shifts and polarization properties of the emission lines can be done based on Fig. 9. Here the magnetic field dependence of the PL peak positions is plotted, where the symbol size represents the PL peak intensity. $\mathrm{T}^{+}$ and $\mathrm{T}^{-}$behave qualitatively differently: First, their singlet states have opposite polarization. It is interesting that the $\mathrm{T}_{s}^{-}$ component with the highest intensity is the one largest in energy. This is typical for trion complexes that leave spin polarized resident carriers after recombination. Second, the triplet state is clearly visible for $\mathrm{T}^{-}$in magnetic fields above $5 \mathrm{~T}$, but it is absent for $\mathrm{T}^{+}$in the field range up to $15 \mathrm{~T}$.

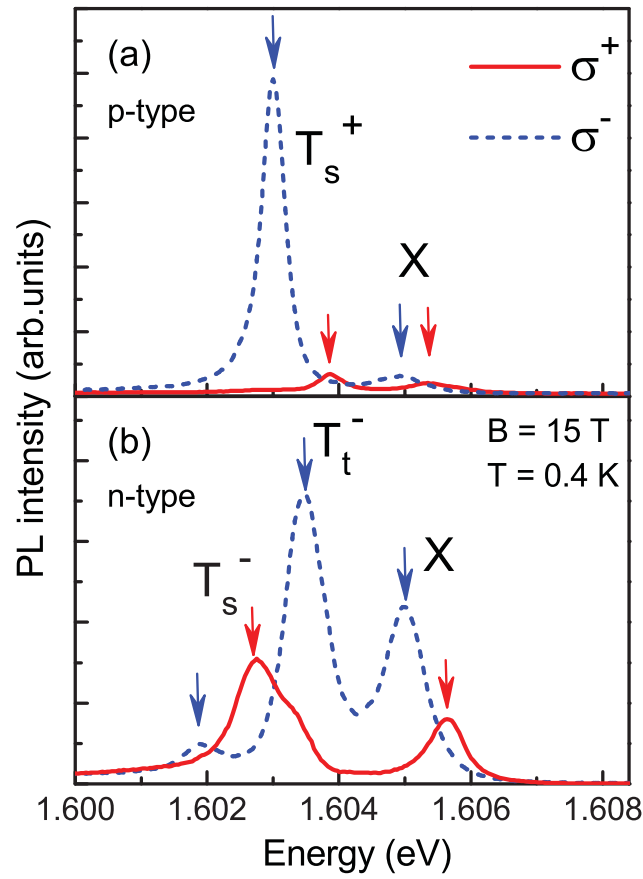

FIG. 8. (Color online) Photoluminescence spectra in the $p$-type and $n$-type regimes, measured under excitation with (a) $1.664 \mathrm{eV}$ and (b) $2.33 \mathrm{eV}$.



FIG. 9. Fan chart of excitons (circles), singlet trions (triangles), and triplet trions (diamonds) extracted from photoluminescence spectra. The circular polarization of the lines is shown by closed $\left(\sigma^{+}\right)$and open $\left(\sigma^{-}\right)$symbols. The symbol size indicates the peak intensity. (a) Below-barrier excitation at a photon energy of $1.66 \mathrm{eV}$. (b) Above-barrier excitation at a photon energy of $2.33 \mathrm{eV}$. 


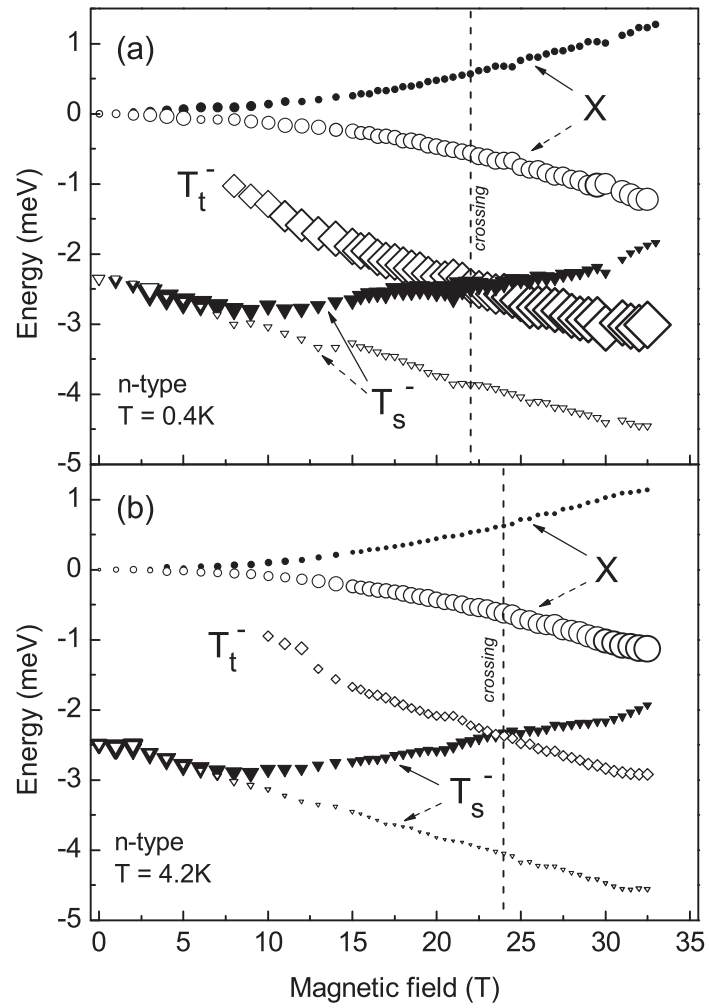

FIG. 10. Energies of exciton and trion optical transitions (the exciton diamagnetic shift is subtracted), measured in the $\mathrm{T}^{-}$regime under laser excitation with a photon energy of $2.33 \mathrm{eV}$. (a) $T=0.4 \mathrm{~K}$. (b) $T=4.2 \mathrm{~K}$. The circular polarization of the lines is shown by closed $\left(\sigma^{+}\right)$and open $\left(\sigma^{-}\right)$symbols.

Also the trion binding energy, seen as the energy separation between the trion and exciton emission lines, behaves differently for $\mathrm{T}^{+}$and $\mathrm{T}^{-}$with increasing magnetic field. This fact is treated in more detail below, where we separate the contributions due to the Coulomb interaction and the Zeeman splitting. The qualitative differences for the $p$-type and $n$-type regimes can be explained based on the trion spin structure schematics given in Sec. III A. To construct them one needs detailed information about the electron and hole Zeeman splittings.

\section{B. Magnetoluminescence of positively and negatively charged trions in magnetic fields up to $33 \mathrm{~T}$}

The application of higher magnetic fields up to $33 \mathrm{~T}$ allows us to reach the regime where the Zeeman splitting energies of excitons and trions become comparable to the trion binding energy. Further, the binding energy of the triplet trion states approaches the singlet binding energy. ${ }^{7}$ The experimental results presented in Figs. 10 and 11 are measured at two temperatures, 0.4 and $4.2 \mathrm{~K}$, which gives us the additional opportunity to identify the PL lines due to the changes of the thermal occupation of the exciton and trion states. For clarity reasons, the exciton diamagnetic shift is subtracted from all these data so that only Zeeman splitting and Coulomb binding energy contributions are left. ${ }^{7}$



FIG. 11. Energies of the exciton and trion optical transitions (the exciton diamagnetic shift is subtracted), measured in the $\mathrm{T}^{+}$regime under laser excitation with a photon energy of $1.66 \mathrm{eV}$. (a) $T=0.4 \mathrm{~K}$. (b) $T=4.2 \mathrm{~K}$. The circular polarization of the lines is shown by closed $\left(\sigma^{+}\right)$and open $\left(\sigma^{-}\right)$symbols. The dashed lines show the expected shift of the $+7 / 2$ to $+3 / 2$ optical transition.

Let us first consider the $n$-type regime presented in Fig. 10 and analyze the behavior of the excitons and singlet trions. One can see that the Zeeman splittings of the exciton and $\mathrm{T}_{s}^{-}$are comparable in size, as predicted by the schematics in Fig. 2. The small difference between the splittings, which is analyzed in more detail in Sec. IV C, is due to a minor deviation in the hole $g$ factors. However, the relative strengths of the $\sigma^{+}$and $\sigma^{-}$components of excitons and singlet trions differ considerably. The exciton emission is $\sigma^{-}$polarized, while $\mathrm{T}_{s}^{-}$ has $\sigma^{+}$polarization. These features are well explained on the basis of the scheme of Fig. 2. For the excitons the Zeeman splitting of the optical transitions is determined by the splitting of the exciton state only. Thus the low energy exciton branch is always the strongest in intensity.

Generally, for the $\mathrm{T}_{s}$ emission, the energy of the optical transitions is composed of the spin splitting of the initial trion state and the splitting of the final free carrier state. However, the sign of its polarization is still defined by the thermal population of the initial state. For the considered case of the $\mathrm{T}_{s}^{-}$emission, this leads to a $\sigma^{+}$polarized PL, although the $\sigma^{+}$transition has a larger photon energy than the $\sigma^{-}$transition.

The triplet trion state $\mathrm{T}_{t}^{-}$is only bound in finite magnetic fields, where its optical transition emerges from the exciton line. In Fig. 10 it appears in $\sigma^{-}$polarization only, and at the lowest temperature measured $(0.4 \mathrm{~K})$ it is the strongest in intensity, even exceeding the intensity of exciton and singlet 
trion lines. In magnetic fields of $20-25 \mathrm{~T}$ the $\mathrm{T}_{t}^{-}$optical transition crosses the oppositely polarized $\mathrm{T}_{s}^{-}$. An intensity exchange between these transitions is seen at $T=0.4 \mathrm{~K}$. Additional details of the triplet trion behavior are discussed below (Sec. IV F). It is worthwhile to note that the bright triplet states, as seen in Ref. 7, are not observed in the present structure, which we explain by the relatively low electron concentration.

The experimental data for the $p$-type regime are displayed in Fig. 11. As expected, the exciton behaves similar to the $n$-type case. Also, the singlet trion $\mathrm{T}_{s}^{+}$has about the same Zeeman splitting as the exciton. Here the trion optical transition with the lowest energy is the strongest in emission intensity, which is consistent with the scheme in Fig. 2. Contrary to $\mathrm{T}^{-}$, no clear triplet state is found for the $\mathrm{T}^{+}$complex. Only in very high magnetic fields, above $28 \mathrm{~T}$, a weak $\sigma^{+}$polarized transition is seen. We assign it to the spin-forbidden optical transition of $\mathrm{T}_{t}^{+}$, as shown in Sec. IV F.

\section{Zeeman splitting and $g$ factors of carriers, excitons, and trions}

In this section we analyze PL and reflectivity experimental data in order to evaluate the Zeeman splittings and $g$-factor values for excitons, electrons, and heavy holes, including their magnetic field dependencies.

In Fig. 12(a) the two spin components of the $1 s$-hh exciton, obtained from the reflectivity spectra, are shown by closed and open circles for $\sigma^{+}$and $\sigma^{-}$polarizations, respectively. The dashed line is the center of gravity of the polarized components; it corresponds to the diamagnetic shift of the exciton energy without the contribution of the Zeeman effect. The magnetic field dependencies of the exciton Zeeman splitting, evaluated from the reflectivity and PL data, are compared in Fig. 12(b). They follow each other very closely showing minor deviations in magnetic fields exceeding $30 \mathrm{~T}$.

The spin splittings of electrons and heavy-holes are plotted in Fig. 12(c) as absolute values. Here the electron dependence is a linear function, corresponding to $\left|g_{e}\right|=1.70$ measured by pump-probe Kerr rotation, see Sec. III B. We assume that the electron $g$ factor is constant in the studied field range, as is shown for CdTe quantum wells in Ref. 40. The heavy-hole dependencies are evaluated from both the Zeeman splitting of the exciton and the splitting of the trion. As introduced in Sec. III A the exciton $g$ factor reads $g_{X}(B)=g_{h}(B)-g_{e}$. Therefore, the heavy-hole spin splitting can be calculated as the difference of the exciton and the electron Zeeman splitting. As one can see from the schematics in Figs. 1 and 2 a similar approach can be applied to obtain the $\mathrm{T}_{s}^{-}$and $\mathrm{T}_{s}^{+}$splittings. As a result, three dependencies for the heavy-hole $g$ factors are shown in Fig. 12(c) being evaluated from the exciton, $\mathrm{T}_{s}^{-}$and $\mathrm{T}_{s}^{+}$Zeeman splittings. We suggest that the deviations between these dependencies reflect different mixing of heavy-hole and light-hole states in the exciton and trion complexes. Detailed analysis requires elaborated calculations accounting for localization and complex valence band structures, which goes beyond the scope of this paper. In the following we will base our analysis on the experimentally obtained

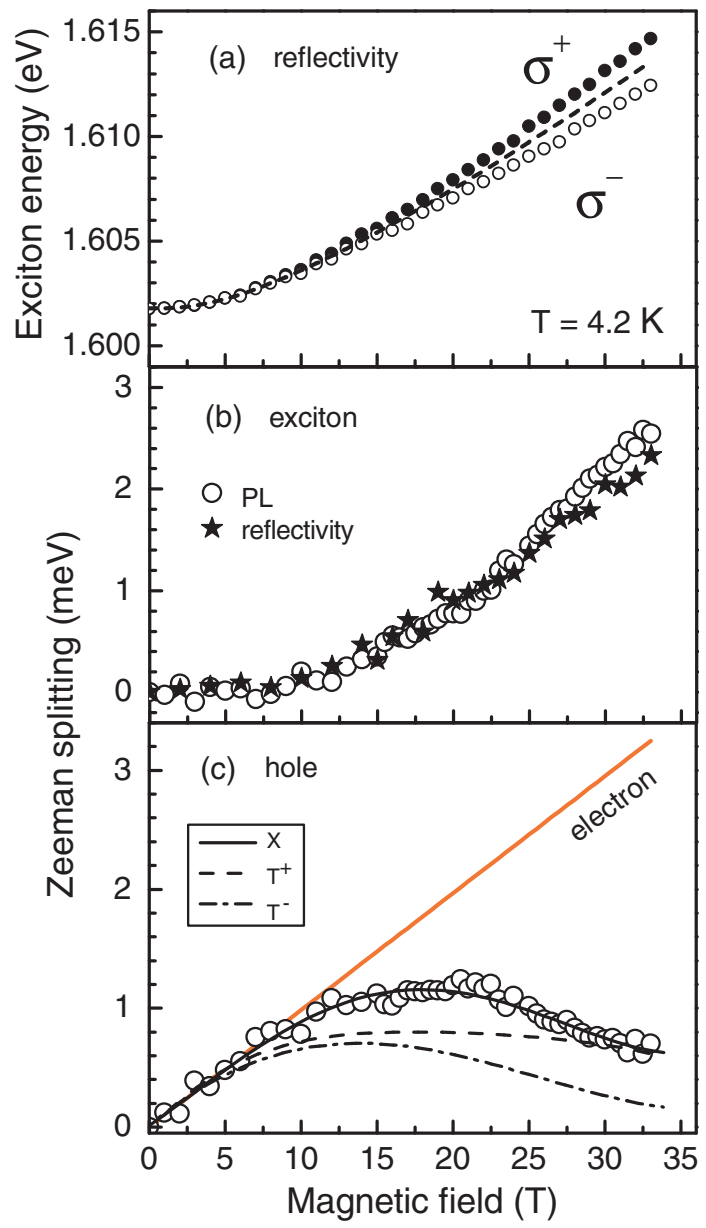

FIG. 12. (Color online) (a) Energy of the $1 s$ heavy-hole exciton in reflectivity spectra as a function of magnetic field. The dashed line shows the center of gravity of the Zeeman split levels. (b) Exciton Zeeman splitting, taken from the photoluminescence (open circles, excitation $1.66 \mathrm{eV}$ ) and reflectivity (solid stars), $T=4.2 \mathrm{~K}$. (c) Zeeman splitting of electrons and heavy holes as a function of the external magnetic field (Faraday geometry). The electron splitting corresponding to $\left|g_{e}\right|=1.70$ is shown by the thick gray (red) line. Results for the heavy holes are evaluated from the Zeeman splittings of excitons, $\mathrm{T}_{s}^{-}$, and $\mathrm{T}_{s}^{+}$. As an example, the hole splitting obtained from the exciton PL (excitation $1.66 \mathrm{eV}$, $T=1.2 \mathrm{~K}$ ), is presented by open circles and a polynomial fit (solid line). For the sake of clarity only interpolations are shown for the other data: dashed line from $\mathrm{T}_{s}^{+} \mathrm{PL}(1.66 \mathrm{eV}, T=0.4 \mathrm{~K})$ and dash-dot line from $\mathrm{T}_{s}^{-} \mathrm{PL}(2.33 \mathrm{eV}, T=1.2 \mathrm{~K})$.

dependencies of $\Delta E_{h}(B)$ for the respective type of exciton and trion.

In Fig. 13 we present information on the Zeeman splittings in the form of magnetic field dependencies of the $g$ factors for excitons and carriers. In this plot we account not only for the absolute values, but also for the $g$-factor signs. For the electron the constant $g_{e}=-1.70$ is shown by the thick gray (red) line. Its value is measured by pump-probe Kerr rotation and the negative sign is taken in analogy to the data of bulk CdTe. ${ }^{41}$ Three dependencies for heavy-holes are evaluated from the Zeeman splittings of Fig. 12(c); the styles of the corresponding lines are the same. 


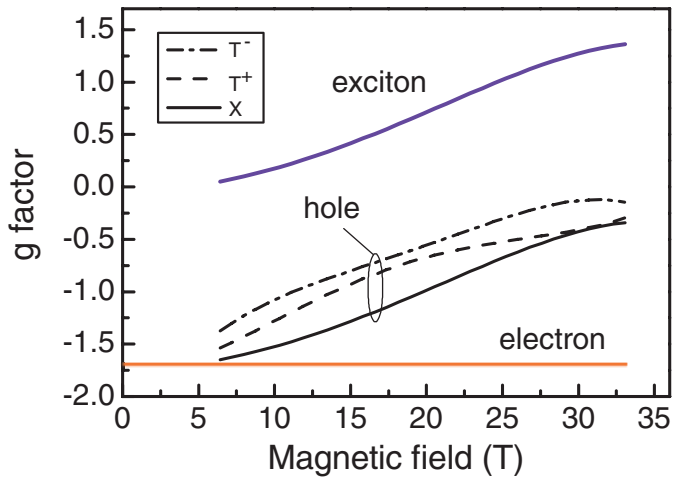

FIG. 13. (Color online) Exciton, electron, and heavy-hole $g$ factors for magnetic fields applied parallel to the structure growth axis (Faraday geometry). For the electron a constant value of $g_{e}=-1.70$ is shown by the thick gray (red) line. For excitons and heavy holes, for the sake of clarity, we show polynomial regressions of the data. Three dependencies are given for the heavy-hole $g$ factors, evaluated from the Zeeman splittings of excitons (solid line), $\mathrm{T}_{s}^{+}$(dashed line), and $\mathrm{T}_{s}^{-}$(dashed-dotted line). For magnetic fields below $6 \mathrm{~T}$ the exciton and hole $g$ factors are not shown as the error of their evaluation becomes too large.

With the information about the electron and hole $g$ factors worked out in this section, we have all the required parameters to reconstruct the spin structure of $\mathrm{T}^{+}$and $\mathrm{T}^{-}$in external magnetic fields. For the studied 20-nm-thick CdTe/Cd ${ }_{0.63} \mathrm{Mg}_{0.37} \mathrm{Te}$ QW the following conditions are fulfilled: $g_{e}<0, g_{h}<0$, and $\left|g_{e}\right|>\left|g_{h}\right|$, and the respective schemes are given in Sec. III A.

\section{Polarization of the trion reflection}

The analysis of polarization characteristics of reflectivity and emission spectra allows one to obtain information on the spin polarization of the resident carriers, excitons, and trions. ${ }^{38,42}$ First we will discuss experimental results on the polarization of reflectivity spectra, which coincide with the polarization of absorption.

In the inset of Fig. 6 the magnetic field dependence of the $\mathrm{T}^{+}$ circular polarization, taken from reflectivity spectra, is shown. It is calculated by $P_{c}^{R}=\left(R^{+}-R^{-}\right) /\left(R^{+}+R^{-}\right)$, where $R^{+}$ and $R^{-}$are the amplitudes of the $\mathrm{T}_{s}^{+}$resonances in $\sigma^{+}$and $\sigma^{-}$ polarizations, respectively. As one can see in Fig. 1 , the $\mathrm{T}_{s}^{+}$ polarization in absorption reflects the polarization of resident holes, which should provide a dominating $\sigma^{-}$polarization. Experimental results from the inset of Fig. 6 are in good agreement with that expectation. At weak magnetic fields the polarization degree increases linearly and reaches saturation at a value of 0.9 for fields exceeding $2 \mathrm{~T}$. The dashed line shows a fit according to the equation for the thermal equilibrium population of Zeeman sublevels:

$$
\left|P_{c}(B)\right|=\tanh \left(\frac{\left|\Delta E_{Z}\right|}{2 k_{B} T}\right) .
$$

Here $k_{B}$ is the Boltzmann constant and $\left|\Delta E_{Z}\right|$ is the Zeeman splitting value. $\left|\Delta E_{Z}(B)\right|$ can be taken from Fig. 12(c). The sign of $P_{c}(B)$ should be selected in accordance with the spin selection rules, shown in Figs. 1 and 2. For the temperature $T$ in Eq. (5) typically the bath temperature of the lattice is taken, while for moderate photoexcitation densities the trions

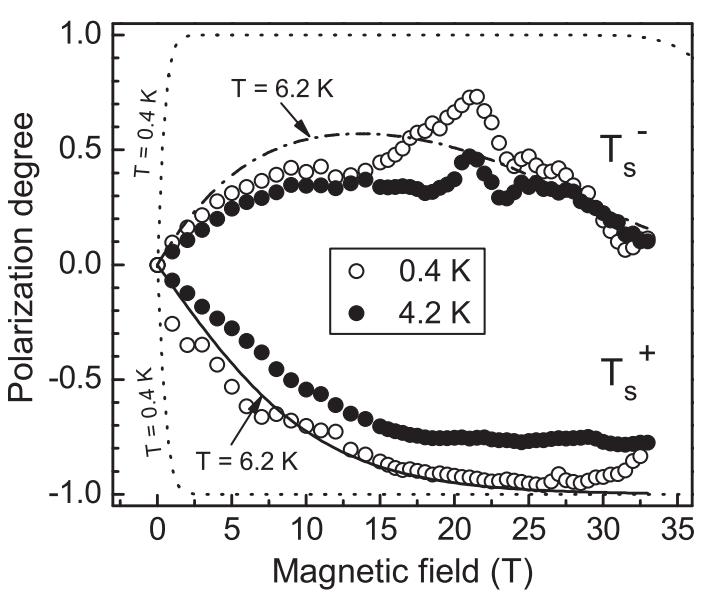

FIG. 14. Circular polarization degree of the photoluminescence, measured for temperatures of $4.2 \mathrm{~K}$ (open symbols) and $0.4 \mathrm{~K}$ (solid symbols). The solid and dash-dot lines show $\mathrm{T}^{+}$and $\mathrm{T}^{-}$fittings by Eq. (5), respectively. The dashed lines represent the degree of polarization, extracted from the same formula for $T=0.4 \mathrm{~K}$.

and resident carriers can be heated and their temperatures can exceed the bath value. In this case the trion temperature can be evaluated as a fitting parameter for the best coincidence with the experimental data. For the results shown in the inset of Fig. 6 the hole temperature of $T_{h}=0.6 \mathrm{~K}$ is obtained from the best fit. It only slightly exceeds the nominal bath temperature in the cryostat of $0.4 \mathrm{~K}$. Such carrier heating under photoexcitation is typical for systems with carrier gases of low density.

\section{E. Polarization of the trion emission}

As one can see in the examples of the PL spectra in Fig. 8 and in the symbol sizes of the fan chart diagrams of Figs. 9, 10, and 11 , the trion emission is strongly polarized. This circular polarization, $P_{c}(B)$, is caused by thermalization of trions on the split Zeeman levels in external magnetic fields. Its magnetic field dependencies for $\mathrm{T}_{s}^{-}$and $\mathrm{T}_{s}^{+}$are shown in Fig. 14 by open and closed symbols for temperatures of 0.4 and $4.2 \mathrm{~K}$, respectively. $P_{c}(B)$ is commonly described by

$$
P_{c}(B)=\frac{I^{+}-I^{-}}{I^{+}+I^{-}},
$$

where $I^{+}$and $I^{-}$are the intensities in $\sigma^{+}$and $\sigma^{-}$polarizations, respectively.

In agreement with Fig. 2, the polarization of the $\mathrm{T}_{s}^{+}$ emission is negative. For both temperatures its value increases with increasing fields and saturates for magnetic fields above $20 \mathrm{~T}$. For $T=0.4 \mathrm{~K}$ the polarization degree approaches the value of $-100 \%$. The polarization dependence for $T=4.2 \mathrm{~K}$ shows smaller values due to the larger thermal energy of $k_{B} T$. A fitting by Eq. (5) of the $T=0.4 \mathrm{~K}$ data is shown by a solid line in Fig. 14. The Zeeman splitting, used for this fit, is the electron splitting (see initial state of $\mathrm{T}_{s}^{+}$in Fig. 2), which increases linearly with magnetic field. The fit reveals a trion temperature of $T=6.2 \mathrm{~K}$, significantly higher, than the bath temperature. We suggest two possible explanations for this: Either there is a finite spin relaxation time, which prevents trion thermalization during its lifetime, or the optical 
excitation causes a measurable temperature increase of the resident carriers, which in turn is translated into the trion heating. For comparison the expected polarization degree for thermalized trions at $T=0.4 \mathrm{~K}$ is given by the dashed line.

The polarization of $\mathrm{T}_{s}^{-}$emission has a positive sign, in agreement with the schematics of Fig. 2. However, the magnetic field dependence of its value differs from the one for $\mathrm{T}_{s}^{+}$, as it saturates at a level of $40 \%-50 \%$ and decreases for $B>25 \mathrm{~T}$. The reason therefore is that the Zeeman splitting of the $\mathrm{T}_{s}^{-}$state is controlled by $g_{h}(B)$, which changes strongly with increasing magnetic field, see Fig. 13. We use the hole Zeeman splitting $\left|\Delta E_{Z}(B)\right|$ from Fig. 12(c) to fit the polarization degree by Eq. (5). The result is shown by a dash-dot line in Fig. 14. $k_{B} T$ and the hole Zeeman splitting have comparable values, as the hole Zeeman splitting does not exceed $1 \mathrm{meV}$. Thus the polarization degree does not saturate at $100 \%$ and is even regressive for high fields due to the nonlinearity of the heavy-hole Zeeman splitting. The estimated trion temperature for $\mathrm{T}_{s}^{-}$of $T=6.2 \mathrm{~K}$ coincides well with the one for the $\mathrm{T}_{s}^{+}$case. Around $B=21 \mathrm{~T}$ the $\mathrm{T}_{s}^{-}$ polarization shows a resonant increase within a rather small field range of about $5 \mathrm{~T}$, which can be assigned to the crossing of $\mathrm{T}_{t}^{-}$and $\mathrm{T}_{s}^{-}$states, see Sec. IV F.

\section{F. Trion triplet states}

In triplet trion states the two identical particles, electrons in $\mathrm{T}_{t}^{-}$and holes in $\mathrm{T}_{t}^{+}$, have the maximal possible total spin with three possible projections. That means for electrons $S=1$ and $S_{z}=0, \pm 1$ and for heavy holes $J=3$ and $J_{z}=0, \pm 3$. In semiconductor QWs, as well as in bulk, the triplet trion states are unbound at zero magnetic field. However, at finite external magnetic fields they are bound, depending on the dimensionality and details of the confinement, such as the particle effective masses and the well widths and depths. For typical QW structures, the corresponding magnetic fields are in the range of a few teslas, $B \sim 2-6 \mathrm{~T}$. Above such fields, the triplet trion states show up in optical emission (PL) spectra as a line below the neutral exciton resonance. ${ }^{7,11,12,20}$

Besides the aforementioned spin quantum numbers, charged trion states in a magnetic field are characterized by a set of two orbital quantum numbers. These are the total angular momentum projection $M_{z}$ and a discrete oscillator quantum number, $k=0,1, \ldots$. The latter is an analog of the centerof-mass total linear momentum, which becomes discrete for a charged complex in a magnetic field. Symmetry considerations show that the trion optical transitions must satisfy the selection rule $\Delta k=0$, reflecting zero linear momentum of the photon (in the dipole approximation), and $\Delta M_{z}= \pm 1$ for the two circular polarizations coming from the angular momentum of the photon. The combination of the selection rules leads to rather stringent limitations for the optical transitions of trions in magnetic fields. Because of this, the bound singlet trion state is "bright" and there exist two types of triplet bound states: a stronger bound "dark" state and a higher lying "bright" state. ${ }^{16}$ The term "dark" means that the corresponding transition is forbidden due to the orbital motion of a charged complex as a whole. More details can be found in Refs. 7, 14 and 15. The dark triplet states have larger binding energies compared with the bright triplets and, therefore, should be

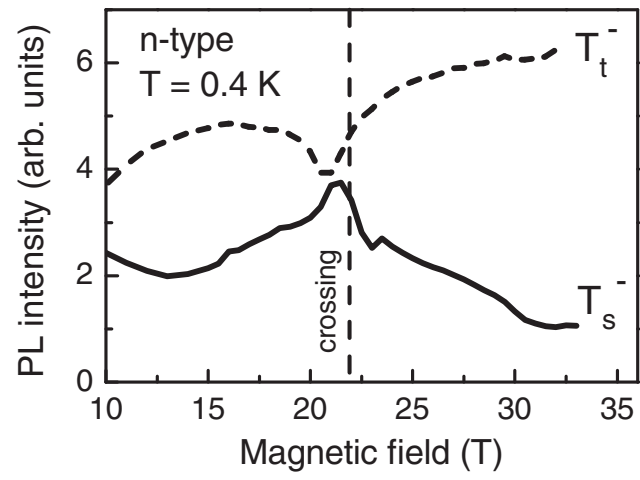

FIG. 15. PL intensities of $\mathrm{T}_{s}^{-}$and $\mathrm{T}_{t}^{-}$in the $n$-type regime, plotted in the vicinity of the singlet-triplet crossing. The vertical dashed line corresponds to the magnetic field where the optical transitions of $\mathrm{T}_{s}^{-}$ and $\mathrm{T}_{t}^{-}$cross each other, see Fig. 10(a).

preferably populated. All the triplet states we discuss in this paper are the dark states. Note that in realistic systems the dark states may acquire finite optical oscillator strengths because of the disorder and impurity scattering ${ }^{15,43}$ and due to electron-electron scattering in the presence of background free carriers. ${ }^{44}$ Combined with large population of the ground state, these symmetry-breaking effects make the nominally dark states visible in the PL spectra, but not in reflectivity spectra.

In the $n$-type regime the $\mathrm{T}_{t}^{-}$emission is clearly observable in a wide range of magnetic fields of 8-33 $\mathrm{T}$, as presented in Fig. 10. This line is strong in intensity and is $\sigma^{-}$circular polarized. Based on the schematics of the trion optical transitions in Fig. 3 we assign this line to the $-1 / 2 \rightarrow+1 / 2$ transition. In magnetic fields around $23 \mathrm{~T}$ the $\mathrm{T}_{t}^{-}$line crosses the $\mathrm{T}_{s}^{-}$line, which is $\sigma^{+}$polarized and corresponds to the transition from $+3 / 2 \rightarrow+1 / 2$, see also Fig. 2 . These $\mathrm{T}_{t}^{-}$ and $\mathrm{T}_{s}^{-}$emission lines have the same final state, namely the $+1 / 2$ resident electron. Therefore, the crossing of their optical transition energies corresponds to the crossing of the triplet state with spin $-1 / 2$ with the singlet state with spin $+3 / 2$. The crossing is accompanied by resonant changes in the emission amplitudes, shown in Fig. 15. The triplet intensity decreases, while the singlet intensity increases simultaneously in the vicinity of the crossing field. This can be explained as an enhanced scattering between triplet and singlet states when they coincide in energy. In this case an additional phonon emission or absorption is not required to assist this transition.

It is interesting to note that in Ref. 7 a singlet-triplet crossover has been already observed in a 12-nm-thick $\mathrm{CdTe} / \mathrm{Cd}_{0.85} \mathrm{Mg}_{0.15} \mathrm{Te} \mathrm{QW}$ with a different set of $g$ factors: $g_{e}<0, g_{h}>0$, and $\left|g_{e}\right|>\left|g_{h}\right|$. The main difference to the 20-nm-thick QW structure, studied in the present paper, is the positive sign of the hole $g$ factor. ${ }^{45}$ In that case the singlet-triplet crossing is hidden in such a way that the singlet and triplet emission lines do not cross each other. However the initial states cross, which can only be recognized by the changes in photoluminescence intensity.

Let us turn to the appearance of $\mathrm{T}_{t}^{+}$triplet states in the $p$-doped regime. Here the experimental results differ significantly from the $n$-doped regime, compare Figs. 10 and 11. No lines associated with $\mathrm{T}_{t}^{+}$can be found in the emission 
spectra for $B<28$ T. As one can see in the schematics of Fig. 3 for $\mathrm{T}_{t}^{+}$, the strongest populated trion state which provides spin-allowed transitions is $+1 / 2$. This emission line $(+1 / 2 \rightarrow+3 / 2)$ should be $\sigma^{-}$polarized. We do not observe it, because most probably this line is underneath the exciton peak. Its Zeeman splitting coincides with the exciton one and the $\mathrm{T}_{t}^{+}$Coulomb binding energy is expected to be close to zero. In this context it is interesting that the exciton line, shown in Fig. 11, becomes significantly stronger for $B>25 \mathrm{~T}$, which may reflect the increasing contribution of the $\mathrm{T}_{t}^{+}$to the exciton peak.

The $\mathrm{T}_{t}^{+}$state with lowest energy has a spin of $+7 / 2$ and its radiative decay to the $\pm 3 / 2$ states is spin forbidden, see Fig. 3. However, the weak $\sigma^{+}$polarized line, appearing in Fig. 11 in magnetic fields above $28 \mathrm{~T}$, can be assigned to the spin-forbidden transition $+7 / 2 \rightarrow+3 / 2$. This assignment is based on its energy position, which is in agreement with the expected shift plotted by the dashed line. The best visibility of this transition we achieve for a data set measured at $T=1.2$ $\mathrm{K}$, using direct optical excitation without fibers, which results in more narrow lines (see Fig. 16). In this case the triplet line is observed in a wider magnetic field range of 20-31 T and in both circular polarizations, which is expectable for spin-forbidden transitions. The lines in the figure trace the expected shift of the $+7 / 2 \rightarrow+3 / 2$ optical transition with the magnetic field dependencies for $g_{h}(B)$ taken from X (solid line) and $\mathrm{T}_{s}^{+}$(dashed line) Zeeman splittings.

In order to assure our identification of the spin-forbidden $\mathrm{T}_{t}^{+}$transition we extend the magnetic field range up to 51.4 T, which allows better spectral separation of the emission lines. Experiments are performed in pulsed magnetic fields and at a temperature of $1.2 \mathrm{~K}$. As one can see in Fig. 17, where the PL spectra are shown for $B=51.4 \mathrm{~T}$ for both circular polarizations, the $\mathrm{T}_{t}^{+}$line is sufficiently strong in intensity and spectrally well separated from the $\mathrm{T}_{s}^{+}$and the $\mathrm{X}$ lines.

Figure 18 shows a comparison of PL and PLE spectra measured at $B=30 \mathrm{~T}$. Three well-resolved lines, identified as $\mathrm{T}_{t}^{+}, \mathrm{T}_{s}^{+}$, and $\mathrm{X}$, are seen in the PL spectrum, detected in $\sigma^{+}$ polarization. The $\mathrm{T}_{t}^{+}$line is the strongest among them. In the PLE spectrum, excited with $\sigma^{+}$polarized light and detected in $\sigma^{-}$polarization, in order to suppress the scattered light of the laser, only two peaks can be well resolved. The energies of these $\mathrm{T}_{s}^{+}$and $\mathrm{X}$ peaks coincide well with the energies of the respective PL lines. However, no PLE peak is found at the position of the $\mathrm{T}_{t}^{+}$emission line. This agrees well with our interpretation of this line as the $+7 / 2 \rightarrow+3 / 2$ optical transition, which should not be seen in absorption or in the PLE spectrum, as it is dipole forbidden and also belongs to the dark triplet states. The fact that it is visible in photoluminescence nevertheless is related to its strongly populated initial state.

\section{G. Coulomb binding energy of the trion states}

The binding energies of the trion ground and excited states result from the Coulomb interaction of three carriers. Theoretically the trion binding energies $E_{b}^{T}$ are calculated as the energy difference of the given trion state $\left(E^{T}\right)$ with respect to the energy of the neutral exciton $\left(E^{X}\right)$ and a free electron (the latter may be chosen as zero energy); i.e., $E_{b}^{T}=E^{X}-E^{T}$.
Thus the energy difference between the optical transitions of the exciton and trion states, measured in emission, absorption, or reflection spectra, can be used to obtain the trion binding energy experimentally. This works well at zero magnetic field, while more care should be taken for extracting data in finite magnetic fields because of the Zeeman energies, as pointed out in the following. The energy dependence of the optical transitions of the trions in magnetic fields has three contributions:

(1) The diamagnetic shift, typical for exciton complexes. Usually, it is equal to that of neutral excitons ${ }^{7,13}$ and its contribution can be accounted for by subtraction of the exciton diamagnetic shift (shift of the center of gravity of the exciton Zeeman doublet) from the trion field dependence.

(2) Variation of the Coulomb binding energy caused by a modification of the wave function by the magnetic field. This is especially important for excited trion states, e.g., triplet states, which are unbound at zero field and stabilized only due to the external field. In addition, the singlet trion state changes its energy in magnetic fields. It is shown theoretically that the magnetic field effect provides a faster growth of the triplet binding energy than for the singlet energy. In the limit of very strong magnetic fields the triplet is the ground state., ${ }^{7,46}$

(3) The Zeeman energy splitting, which results, in the case of trions, from both the splittings of the trion state and of the resident carrier left behind after the recombination (Figs. 2 and $3)$. In the case when $g_{e}(B)$ and $g_{h}(B)$ are identical for excitons and trions, their Zeeman splittings are equal. In this case, the Zeeman contribution can be simply extracted by taking the difference between the exciton and trion lines, measured in the same circular polarization. This approach is often used to extract the Coulomb binding energy of singlet trion states. Still one should be careful here as, e.g., in the structures studied in this paper, $g_{h}(B)$ dependencies are not the same for excitons and trions, see Figs. 13 and 12(c). For the triplet states this simplified approach can be valid in rare cases only and the procedure developed in Ref. 7, which is based on detailed experimental information of the Zeeman splittings of excitons, trions, and carriers, should be used. We apply this procedure to evaluate the Coulomb binding energies of the singlet and triplet state in our sample.

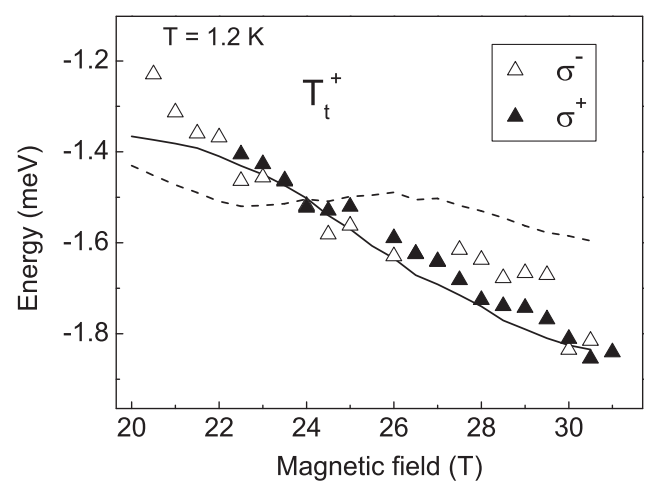

FIG. 16. $\mathrm{T}_{t}^{+}$energies (diamagnetic shift subtracted) plotted against the magnetic field. Besides the $\sigma^{+}$features (solid symbols) a $\sigma^{-}$contribution is also visible (open symbols). Solid and dashed lines show the calculated $+7 / 2 \rightarrow+3 / 2$ optical transition of $\mathrm{T}_{t}^{+}$, based on $\mathrm{X}$ and $\mathrm{T}_{s}^{+}$Zeeman splittings, respectively. 


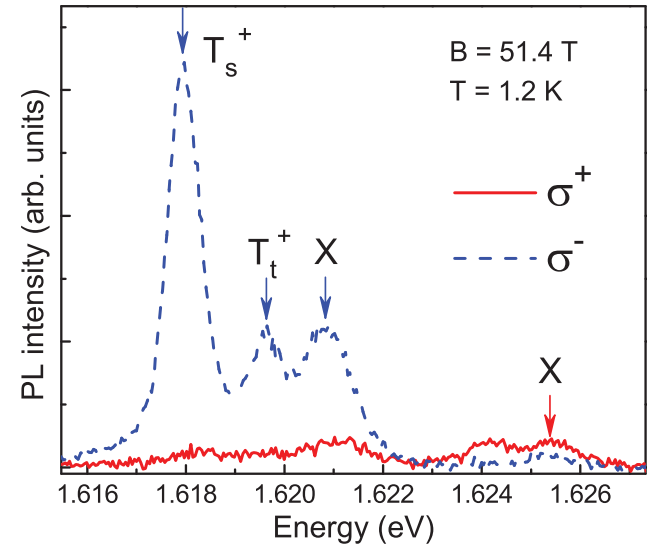

FIG. 17. (Color online) Photoluminescence spectra for two circular polarizations at a pulsed magnetic field of $51.4 \mathrm{~T}$ in the $p$-type regime. In the $\sigma^{-}$polarization $\mathrm{X}$ and $\mathrm{T}_{t}^{+}$are clearly seen as two separate peaks.

The results for the $\mathrm{T}^{-}$and $\mathrm{T}^{+}$singlet and $\mathrm{T}^{-}$triplet states are collected in Fig. 19. Note that due to the tunability of the resident carrier type in the studied 20-nmthick $\mathrm{CdTe} / \mathrm{Cd}_{0.63} \mathrm{Mg}_{0.37} \mathrm{Te} \mathrm{QW}$, these trion Coulomb energies are measured in the very same structure, i.e., for identical confinement and localization conditions. The results measured at two different temperatures of 0.4 and $4.2 \mathrm{~K}$ are shown in Fig. 19(a) by closed and open circles, respectively. No significant difference between those two temperatures is visible. However, a qualitatively different behavior of the positively and negatively charged singlet trions is immediately obvious.

In the $n$-type regime the $\mathrm{T}_{s}^{-}$binding energy at $B=0 \mathrm{~T}$ is $2.4 \mathrm{meV}$ and increases up to $3.2 \mathrm{meV}$ at $33 \mathrm{~T}$. The $\mathrm{T}_{s}^{+}$ binding energy in the $p$-type regime starts from $2.2 \mathrm{meV}$ at $B=0 \mathrm{~T}$ and decreases with growing magnetic field until it reaches its minimal value of $1.7 \mathrm{meV}$ at $16 \mathrm{~T}$. After that it starts to increase again slightly, approaching $2.0 \mathrm{meV}$ at $33 \mathrm{~T}$. In the pulsed fields the $\mathrm{T}_{s}^{+}$binding energy keeps increasing and reaches $2.8 \mathrm{meV}$ at $51.4 \mathrm{~T}$, see Fig. 20 .

The qualitative difference in the magnetic field dependencies of the $\mathrm{T}_{s}^{-}$and $\mathrm{T}_{s}^{+}$binding energies is also reported for GaAs- and ZnSe-based QWs. ${ }^{10,12}$ This may be related to

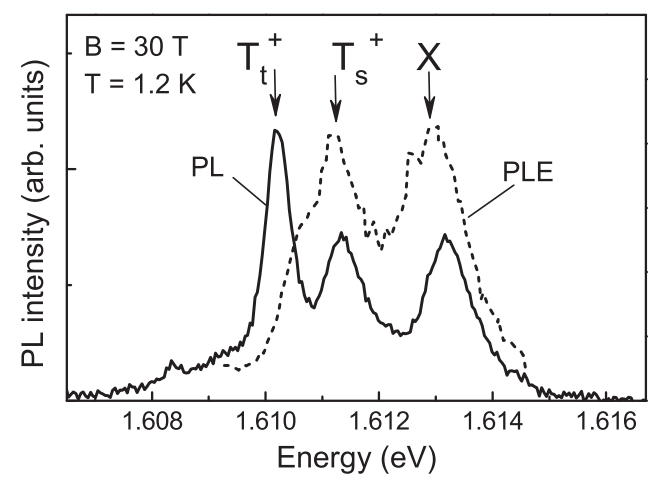

FIG. 18. Photoluminescence (solid line) and PL excitation (dashed line) spectra at $B=30 \mathrm{~T}$ in the $p$-type regime. The PL is detected in $\sigma^{+}$polarization. The PLE is excited with a $\sigma^{+}$polarized laser and detected at $1.609 \mathrm{eV}$ in $\sigma^{-}$polarization.



FIG. 19. Magnetic field dependencies of (a) Coulomb binding energies of positively and negatively charged singlet trions, $T=4.2$ $\mathrm{K}$ (open symbols) and $0.4 \mathrm{~K}$ (closed symbols). (b) Coulomb binding energy of $\mathrm{T}^{-}$singlet and triplet states.

the different structure of the $\mathrm{T}^{+}$and $\mathrm{T}^{-}$wave functions. ${ }^{28}$ Qualitatively, the $\mathrm{T}^{-}$is a complex with one much heavier hole acting as a center with two electrons orbiting around it. The atomic physics analog of $\mathrm{T}^{-}$is the negative hydrogen ion $\mathrm{H}^{-}$, well studied for its important role in astrophysics. With increasing magnetic fields the electron probability distribution of $\mathrm{T}^{-}$is squeezed, as a result of the reducing magnetic length. This increases the overlap with the hole, so that the mean electron-hole distance becomes smaller and thus the $\mathrm{T}^{-}$binding energy increases with $B$. For the $\mathrm{T}^{+}$, however, we have two holes as two centers with the one electron in between them. The atomic physics analog of $\mathrm{T}^{+}$is the $2 \mathrm{D}$ molecular positive ion $\mathrm{H}_{2}^{+}$consisting of two heavy protons and one much lighter electron mediating the chemical bond. With increasing magnetic field the width of the electron probability distribution decreases (with the magnetic length), but the average separation between the positive centers does not exhibit a substantial variation, as the electron remains in between the centers. Therefore, the $\mathrm{T}^{+}$binding energy does not change appreciably with $B$. Based on this, one should expect rather different behaviors for negatively and positively charged trions, which may explain the experimental findings. Note, however, that here we are dealing with interparticle correlations in a spatially confined quantum mechanical system consisting of a few particles. An additional complication comes from the presence of the external magnetic field, for which barely any simple and intuitively clear picture can catch all the qualitative aspects and dependencies.

Additionally, the singlet and triplet states of $\mathrm{T}^{-}$are compared in Fig. 19(b). The $\mathrm{T}_{t}^{-}$appears in the spectra, i.e., becomes a bound state, around $B=5 \mathrm{~T}$ and its binding energy increases significantly up to $1.9 \mathrm{meV}$ with growing field strength showing a saturation at this level. It is interesting that the experimental behavior in 12-nm-thick CdTe-based QWs is 


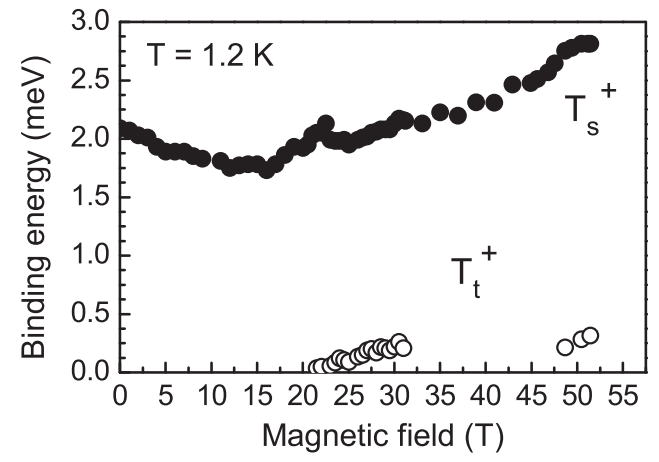

FIG. 20. Coulomb binding energies of $\mathrm{T}^{+}$trion states in the $p$ type regime up to $B=51 \mathrm{~T}$. Data for the singlet and triplet trions are shown by solid and open symbols, respectively.

different, see Fig. 3(c) in Ref. 7, where the triplet shift increases continuously with a tendency to reach the binding energy of the singlet state. This difference may be tentatively attributed to the change in the regime from a quasi-2D to quasi-3D that occurs for a wider 20-nm-thick QW in strong magnetic fields.

In the $p$-type regime the triplet state of the positively charged trion is identified. Due to the large contribution of the Zeeman splitting to the energy shift of the $\mathrm{T}_{t}^{+}$emission line, it becomes resolvable in magnetic fields exceeding $22 \mathrm{~T}$, as one can see in Figs. 11 and 16. The Coulomb binding energy of the $\mathrm{T}_{t}^{+}$state is shown in Fig. 20 by open circles. It is considerably smaller than the $\mathrm{T}_{t}^{-}$binding energy, not exceeding $0.3 \mathrm{meV}$ even in magnetic fields as strong as $50 \mathrm{~T}$.

\section{CONCLUSIONS}

A comparative study of the energy and spin structure of negatively versus positively charged excitons is performed for a 20 -nm-thick $\mathrm{CdTe} /(\mathrm{Cd}, \mathrm{Mg}) \mathrm{Te}$ quantum well in high magnetic fields. Tuning between the $p$ - and $n$-type regimes of resident carriers of low density is realized optically by an additional above-barrier illumination. The measured binding energies of the $\mathrm{T}^{-}$and $\mathrm{T}^{+}$singlet trion states demonstrate qualitatively different magnetic field dependencies. Namely, while the $\mathrm{T}_{s}^{-}$binding energy increases, the $\mathrm{T}_{s}^{+}$binding energy decreases with increasing $B$. Polarizations and Zeeman splittings of all the identified photoluminescence lines are in qualitative agreement with the suggested spin structure schematics. The dark triplet trion states are observed both for the negative and positive excess carrier regimes. In the $n$-type regime a change in the ground state from singlet to dark triplet is observed. In the $p$-type regime a spin-forbidden triplet recombination is found, being visible due to the large population of the initial state and due to weak spin-symmetrybreaking effects present in the $\mathrm{CdTe} /(\mathrm{Cd}, \mathrm{Mg}) \mathrm{Te}$ quantum well.

\section{ACKNOWLEDGMENTS}

The work was supported by the Deutsche Forschungsgemeinschaft, the EU Seventh Framework Programme (Grant No. 237252, Spin-optronics), and the Russian Foundation for Basic Research. Research in Poland was partially supported by the European Union within European Regional Development Fund, through grant Innovative Economy (POIG.01.01.02-00108/09). Experiments in high magnetic fields performed in Nijmegen were partly sponsored by DeNUF and EuroMagNET II under the EU Contract No. 228043, and were part of the research program of the Stichting voor Fundamenteel Onderzoek der Materie (FOM), which is financially supported by the Nederlandse Organisatie voor Wetenschappelijk Onderzoek (NWO).
${ }^{1}$ M. A. Lampert, Phys. Rev. Lett. 1, 450 (1958).

${ }^{2}$ K. Kheng, R. T. Cox, Y. Merle d'Aubigne, F. Bassani, K. Saminadayar, and S. Tatarenko, Phys. Rev. Lett. 71, 1752 (1993).

${ }^{3}$ G. Finkelstein, H. Shtrikman, and I. Bar-Joseph, Phys. Rev. Lett. 74, 976 (1995).

${ }^{4}$ V. V. Solovyev and I. V. Kukushkin, Phys. Rev. B 79, 233306 (2009).

${ }^{5}$ G. Finkelstein, H. Shtrikman, and I. Bar-Joseph, Phys. Rev. B 53, R1709 (1996).

${ }^{6}$ R. T. Cox, V. Huard, K. Kheng, S. Lovisa, R. B. Miller, K. Saminadayar, A. Arnoult, J. Cibert, and S. Tatarenko, Acta Phys. Pol. A 94, 99 (1998).

${ }^{7}$ G. V. Astakhov, D. R. Yakovlev, V. V. Rudenkov, P. C. M. Christianen, T. Barrick, S. A. Crooker, A. B. Dzyubenko, W. Ossau, J. C. Maan, G. Karczewski, and T. Wojtowicz, Phys. Rev. B 71, 201312R (2005).

${ }^{8}$ G. V. Astakhov, D. R. Yakovlev, V. P. Kochereshko, W. Ossau, J. Nürnberger, W. Faschinger, and G. Landwehr, Phys. Rev. B 60, R8485 (1999).

${ }^{9}$ G. V. Astakhov, V. P. Kochereshko, D. R. Yakovlev, W. Ossau, J. Nürnberger, W. Faschinger, and G. Landwehr, Phys. Rev. B 62, 10345 (2000).
${ }^{10}$ G. V. Astakhov, D. R. Yakovlev, V. P. Kochereshko, W. Ossau, W. Faschinger, J. Puls, F. Henneberger, S. A. Crooker, Q. McCulloch, D. Wolverson, N. A. Gippius, and A. Waag, Phys. Rev. B 65, 165335 (2002).

${ }^{11}$ A. J. Shields, M. Pepper, M. Y. Simmons, and D. A. Ritchie, Phys. Rev. B 52, 7841 (1995).

${ }^{12}$ S. Glasberg, G. Finkelstein, H. Shtrikman, and I. Bar-Joseph, Phys. Rev. B 59, R10425 (1999).

${ }^{13}$ D. R. Yakovlev, G. V. Astakhov, W. Ossau, S. A. Crooker, K. Uchida, N. Miura, A. Waag, N. A. Gippius, A. Yu. Sivachenko, and A. B. Dzyubenko, Phys. Status Solidi 227, 353 (2001).

${ }^{14}$ A. B. Dzyubenko, H. A. Nickel, T. Yeo, B. D. McCombe, and A. Petrou, Phys. Status Solidi B 227, 365 (2001).

${ }^{15}$ A. B. Dzyubenko and A. Yu. Sivachenko, Phys. Rev. Lett. 84, 4429 (2000).

${ }^{16}$ A. Wojs, J. J. Quinn, and P. Hawrylak, Phys. Rev. B 62, 4630 (2000).

${ }^{17}$ A. Wojs, J. J. Quinn, and P. Hawrylak, Physica E (Amsterdam) 8, 254 (2000).

${ }^{18}$ C. Riva, F. M. Peeters, and K. Varga, Phys. Rev. B 63, 115302 (2001). 
${ }^{19}$ D. Andronikov, V. Kochereshko, A. Platonov, T. Barrick, S. A. Crooker, and G. Karczewski, Phys. Rev. B 72, 165339 (2005).

${ }^{20}$ G. Yusa, H. Shtrikman, and I. Bar-Joseph, Phys. Rev. Lett. 87, 216402 (2001).

${ }^{21}$ A. J. Shields, J. L. Osborne, M. Y. Simmons, M. Pepper, and D. A. Ritchie, Phys. Rev. B 52, R5523 (1995).

${ }^{22}$ J. L. Osborne, A. J. Shields, M. Pepper, F. M. Bolton, and D. A. Ritchie, Phys. Rev. B 53, 13002 (1996).

${ }^{23}$ A. S. Bracker, E. A. Stinaff, D. Gammon, M. E. Ware, J. G. Tischler, D. Park, D. Gershoni, A. V. Filinov, M. Bonitz, F. M. Peeters, and C. Riva, Phys. Rev. B 72, 035332 (2005).

${ }^{24}$ L. Bryja, A. Wojs, J. Misiewicz, M. Potemski, D. Reuter, and A. Wieck, Phys. Rev. B 75, 035308 (2007).

${ }^{25}$ A. Haury, A. Arnoult, V. A. Chitta, J. Cibert, Y. Merle d'Aubigne, S. Tatarenko, and A. Wasiela, Superlattices Microstruct. 23, 1097 (1998).

${ }^{26}$ P. Kossacki, J. Cibert, D. Ferrand, Y. Merle d'Aubigne, A. Arnoult, A. Wasiela, S. Tatarenko, and J. A. Gaj, Phys. Rev. B 60, 16018 (1999).

${ }^{27}$ P. Kossacki, J. Phys. Condens. Matter 15, R471 (2003).

${ }^{28}$ R. A. Sergeev and R. A. Suris, Fiz. Tverd. Tela 43, 714 (2001) [Phys. Solid State 43, 746 (2001)].

${ }^{29}$ A. Esser, E. Runge, R. Zimmermann, and W. Langbein, Phys. Rev. B 62, 8232 (2000).

${ }^{30}$ A. Esser (private communication).

${ }^{31}$ Low carrier density means that the Fermi energy of the carrier gas is smaller than the typical localizing potential for the carrier motion in the QW plane. This localizing potential is provided by well width fluctuations and is represented by the exciton broadening of about $0.6 \mathrm{meV}$ in the studied sample.

${ }^{32}$ E. A. Zhukov, D. R. Yakovlev, M. Gerbracht, G. V. Mikhailov, G. Karczewski, T. Wojtowicz, J. Kossut, and M. Bayer, Phys. Rev. B 79, 155318 (2009).

${ }^{33}$ V. Ciulin, P. Kossacki, S. Haacke, J.-D. Ganiere, B. Deveaud, A. Esser, M. Kutrowski, and T. Wojtowicz, Phys. Rev. B 62, R16310 (2000).
${ }^{34}$ D. R. Yakovlev and M. Bayer, in Spin Physics in Semiconductors, edited by M. I. Dyakonov (Springer-Verlag, Berlin, 2008), Ch. 6, p. 135.

${ }^{35}$ E. A. Zhukov, D. R. Yakovlev, M. M. Glazov, L. Fokina, G. Karczewski, T. Wojtowicz, J. Kossut, and M. Bayer, Phys. Rev. B 81, 235320 (2010).

${ }^{36}$ E. A. Zhukov, D. R. Yakovlev, M. Bayer, M. M. Glazov, E. L. Ivchenko, G. Karczewski, T. Wojtowicz, and J. Kossut, Phys. Rev. B 76, 205310 (2007).

${ }^{37}$ I. A. Yugova, A. Greilich, D. R. Yakovlev, A. A. Kiselev, M. Bayer, V. V. Petrov, Y. K. Dolgikh, D. Reuter, and A. D. Wieck, Phys. Rev. B 75, 245302 (2007).

${ }^{38}$ G. V. Astakhov, V. P. Kochereshko, D. R. Yakovlev, W. Ossau, J. Nürnberger, W. Faschinger, G. Landwehr, T. Wojtowicz, G. Karczewski, and J. Kossut, Phys. Rev. B 65, 115310 (2002).

${ }^{39}$ As the $g$ factors discussed here are constructed from the measured Zeeman splitting, they should be formally introduced as effective $g$ factors. For the sake of simplicity, however, we omit the term effective in this article.

${ }^{40}$ H. W. van Kesteren, E. C. Cosman, W. A. J. A. van der Poel, and C. T. Foxon, Phys. Rev. B 41, 5283 (1990).

${ }^{41}$ A. A. Sirenko, T. Ruf, M. Cardona, D. R. Yakovlev, W. Ossau, A. Waag, and G. Landwehr, Phys. Rev. B 56, 2114 (1997).

${ }^{42}$ C. R. L. P. N. Jeukens, P. C. M. Christianen, J. C. Maan, D. R. Yakovlev, W. Ossau, V. P. Kochereshko, T. Wojtowicz, G. Karczewski, and J. Kossut, Phys. Rev. B 66, 235318 (2002).

${ }^{43}$ A. B. Dzyubenko and A. Yu. Sivachenko, Physica E 6, 226 (2000).

${ }^{44}$ D. Sanvitto, D. M. Whittaker, A. J. Shields, M. Y. Simmons, D. A. Ritchie, and M. Pepper, Phys. Rev. Lett. 89, 246805 (2002).

${ }^{45}$ The value and even the sign of the hole $g$ factor in QWs is controlled by structure parameters responsible for mixing of heavy-hole and light-hole states. The mixing is also influenced by external magnetic fields, which results in a field-dependent hole $g$ factor, see Sec. IV C.

${ }^{46}$ D. M. Whittaker and A. J. Shields, Phys. Rev. B 56, 15185 (1997). 